\title{
A Mini Review on the Chemical Compounds of the Genus Acacia
}

\author{
Mohammad Musarraf Hussain \\ Department of Pharmacy, Faculty of Life and Earth Sciences, Jagannath University \\ Dhaka-1100, Bangladesh
}

(Received: May 18, 2019; Accepted: July 11, 2019; Published: July 22, 2019)

\begin{abstract}
The genus Acacia is a pioneering source of diversified chemical compounds. The purpose of this review is to compile of the phytochemicals from few species of Acacia. A total ten species of Acacia were studied and seventy six (1-76) phytoconstituents, including their chemical structures are reported in this review. The highest number of chemical compounds has been reported from Acacia nilotica.
\end{abstract}

Key words: Acacia, Mimosaceae, Leguminosae, Fabaceae, and Chemical compounds.

\section{Introduction}

Nature is a significant resource of medicinal plants and these plants are using as conventional agents for the treatment of various diseases from many years (Hussain 2019, 2018; Hussain et al., 2016a, 2016b). The medicinal plants under Acacia genus is bearing therapeutic properties such as antimicrobial, anti-inflammatory, anti-plasmodial, and cytotoxic activity (Billah et al., 2013; Hussain et al., 2011, 2010, 2008; Ismail et al., 2010). Acacia is a big pantropical genus comprising greater than 1300 species under the family of Mimosoideae and subfamily Fabaceae. A. albida (Family: Momosaceae) is a tree (24 $\mathrm{m}$ in height) bearing big straight bowl and rounded crown. A. catechu (Family: Leguminosae) is a deciduous and thorny tree grows up to $15 \mathrm{~m}$ in height. A. cyclops (Family: Fabaceae) is a small tree or coastal shrub native to Australia. A. kamerunensis (Family: Momosaceae) is a lowland rain secondary forest and scandent shrub (5 $\mathrm{m}$ tall) distributed in Sierra Leone and Uganda. A. mearnsii (Family: Momosaceae, black wattle) is a significant species for tannin production and woodchip factories. A. nilotica (Family: Fabaceae): is a thorny tree up to $15 \mathrm{~m}$ in height with a characteristic necklace appearance. A. pennata
(Family: Mimosaceae) is a perpetual woody climber having bi-pennate leaves.

\section{Chemical compounds}

A total ten species of the genus Acacia have been studied and seventy six (1-76) molecules were reported in this review as phytochemicals. The studied species of Acacia are Acacia albida, A. catechu, A. cochliacantha, A. cyclops, A. kamerunensis, A. mearnsii, A. mollissima A. nilotica, A. oxyphylla and A. pennata.

Acacia albida: Reported triterpene saponins and other compounds from Acacia albida are Albidoside A (1), Albidoside B (2), Albidoside C (3), Albidoside D (4), Albidoside E (5), Albidoside F (6), Albidoside G (7) $\beta$-Amyrin (8), $\beta$-Sitosterol (9), $\beta$-Sitosterol-3$O$ - $\beta$ - $D$-Glucopyranoside (10), Quercetin (11), Gallic acid (12), Rhamnocitrin (13), Afzelin (14), and (6S, 9S)-Roseoside (15), (Figure 1) (Tchoukoua et al., 2017; Mohammed et al., 2018).

Acacia catechu: Reported compounds from this plant are (3R, 4R)-3-(3,4-dihydroxyphenyl)-4hydroxycy-clohexanone (16), (4R)-5-(1-(3,4dihydrophenyl)-3-oxobutyl)-dihydrofuran-2(3H)-one (17), 4-Hyroxybenzoic acid (18), Kaempferol (19), 3,4',7-trihydroxy-3', 5-dimethoxyflavone

(20), 
Catechin (21), Epicatechin (22), Afzelechin (23), consisting alleopathic effect are Mollisside B (35), Epiafzelechin (24), Mesquitol (25), Ophioglonin (Z)-3-hexen-1-ol acetate (36), 4-Oxoisophorone (37), (26), Aromadendrin (27), and Phenol (28) (Figure 2) (Li et al., 2011).

Acacia cyclops: Isolated saponins and other compounds with chemical structure from this plant (Z)- $\beta$-ocimene (38), Cyclopside 1 (39), Cyclopside 2 (40), Nonadecane (41), and Caryophyllene (42) (Figure 4) (Kotze et al., 2010; Jelassi et al., 2014, 2016).
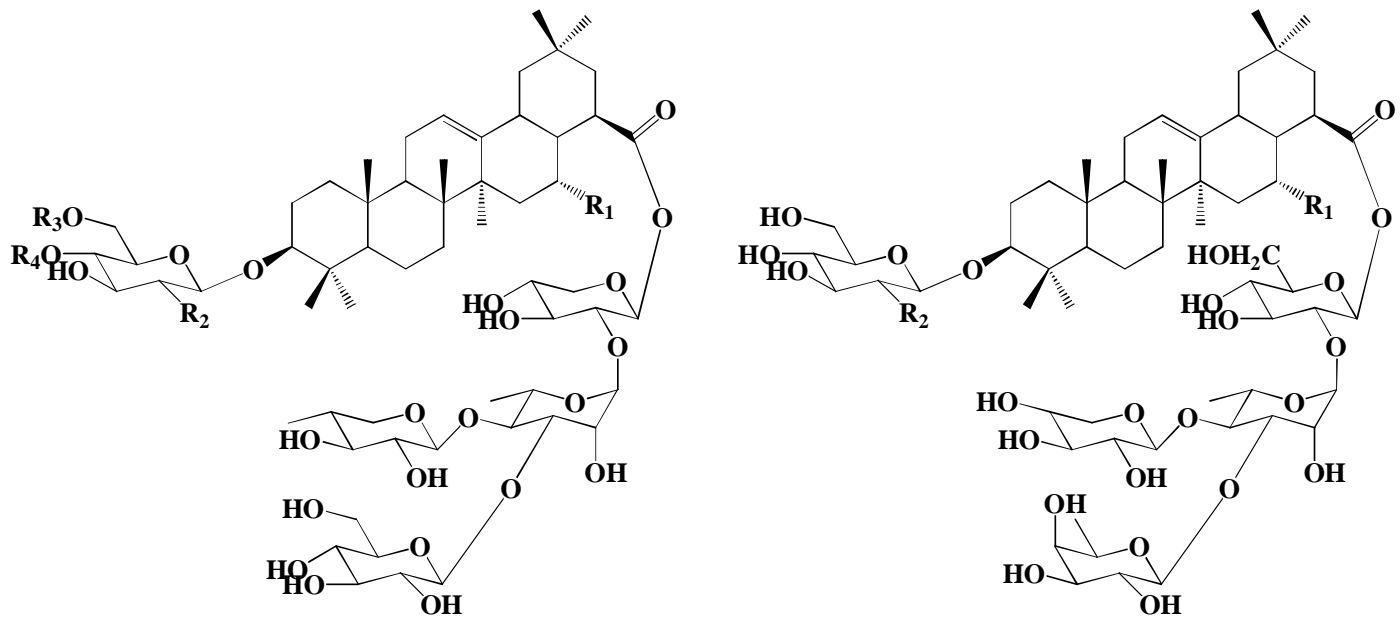

1: $R_{1}=H, R_{2}=$ NHAc, $R_{3}=H, R_{4}=H$

3: $R_{1}=H, R_{2}=$ OGLc, $R_{3}=H, R_{4}=H$

4: $R_{1}=H, R_{2}=$ NHAc, $R_{3}=H, R_{4}=$ GLc

2: $\mathrm{R}_{1}=\mathrm{H}, \mathrm{R}_{2}=$ OGLc

6: $R_{1}=O H, R_{2}=N H A c, R_{3}=H, R_{4}=G L c$

5: $R_{1}=O H, R_{2}=$ NHAc

7: $R_{1}=O H, R_{2}=$ NHAc, $R_{3}=A r a, R_{4}=H$
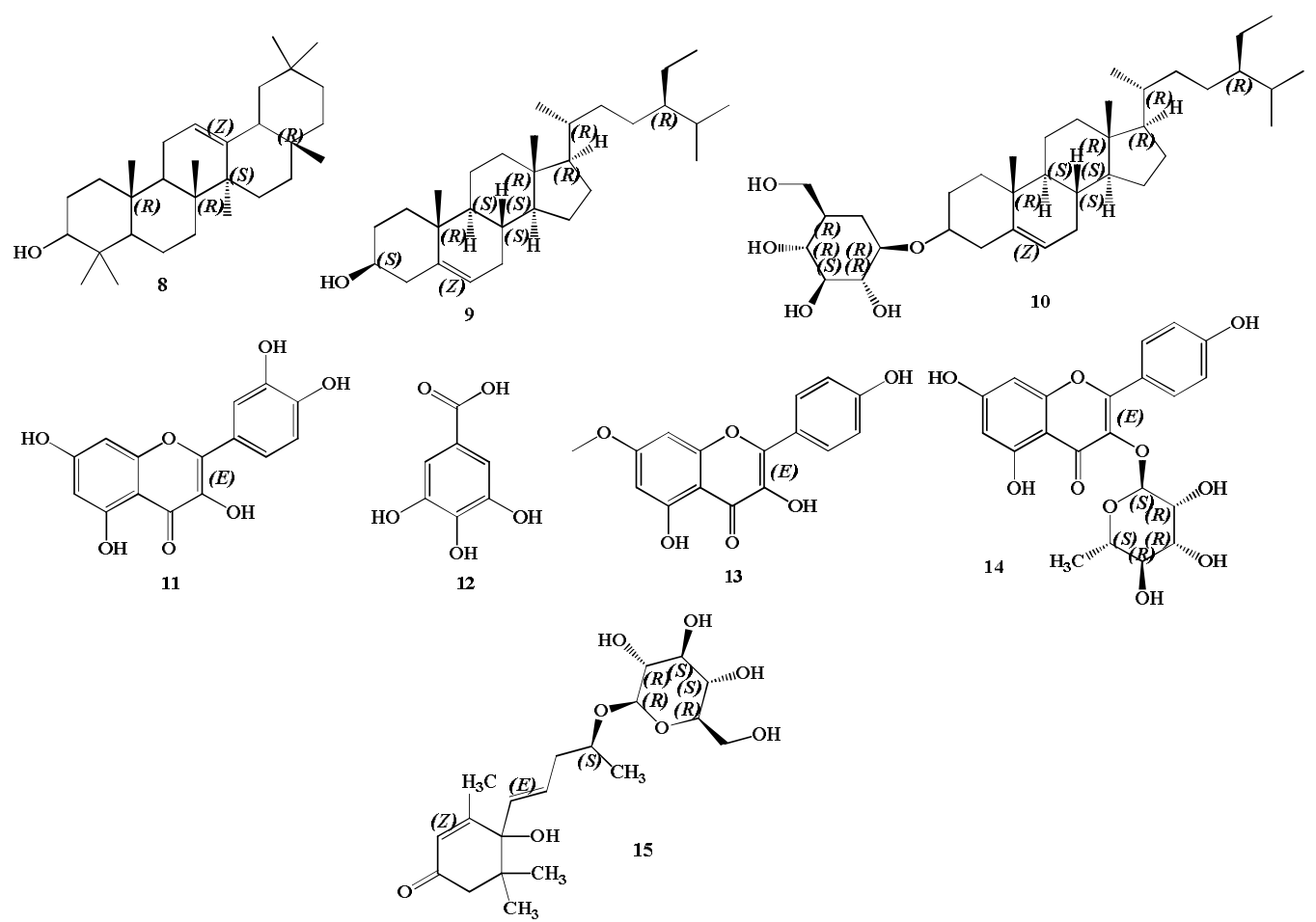

Figure 1. Triterpene saponin and other compounds from Acacia albida. 
<smiles>CC1CCC(=O)CC1c1ccc(O)c(O)c1</smiles>

16<smiles>CC(=O)CC(OC1CCC(=O)O1)c1ccc(O)c(O)c1</smiles>

17<smiles>O=C(O)c1ccc(O)cc1</smiles>

18<smiles>Oc1ccccc1</smiles>

28<smiles>O=c1c(O)c(-c2ccc(O)cc2)oc2cc(O)cc(O)c12</smiles><smiles>Oc1cc(O)c2c(c1)O[C@](O)(c1ccc(O)c(O)c1)C[C@H]2O</smiles><smiles>Oc1ccc([C@@H]2Oc3c(ccc(O)c3O)C[C@H]2O)cc1O</smiles>

25<smiles>COc1cc(OC)cc(-c2oc3cc(O)cc(O)c3c(=O)c2O)c1</smiles><smiles>OC[C@@H](O)[C@]1(c2ccc(O)c(O)c2)Oc2cc(O)cc(O)c2O1</smiles><smiles>Oc1ccc(-c2oc3cc(O)cc(O)c3c2O)cc1</smiles><smiles>Oc1ccc(-c2oc3cc(O)cc(O)c3c2O)cc1</smiles><smiles>O=c1c2c(oc3cc(O)cc(O)c13)-c1ccc(O)c(O)c1CO2</smiles>

26<smiles>O=C1c2c(O)cc(O)cc2O[C](c2ccc(O)cc2)[C@H]1O</smiles>

Figure 2. Compounds from Acacia catechu.

Acacia cochliacantha: Elucidated secondary metabolites having ovicidal activity from this species are Caffeic acid (29), $p$-Coumaric acid (30), Ferulic acid (31), Methyl caffeate (32), Methyl-p-coumarate (33), and Methylferulate (34) (Figure 3) (CastilloMitre et al., 2017).<smiles>[R10]OC(=O)/C=C/c1ccc(O)c([R1])c1</smiles>

29-34

Figure 3. Reported molecules from Acacia cochliacantha.

29: $\mathrm{R}_{1}=O H, \mathrm{R}_{\mathbf{2}}=\mathrm{H}$

30: $\mathrm{R}_{\mathbf{1}}=\mathrm{H}, \mathrm{R}_{\mathbf{2}}=\mathrm{H}$

31: $\mathrm{R}_{\mathbf{1}}=\mathrm{OH}, \mathrm{R}_{\mathbf{2}}=\mathrm{H}$

32: $\mathrm{R}_{1}=\mathrm{OH}, \mathrm{R}_{\mathbf{2}}=\mathrm{CH}_{3}$

33: $\mathrm{R}_{\mathbf{1}}=\mathrm{H}, \mathrm{R}_{\mathbf{2}}=\mathrm{CH}_{3}$

34: $\mathrm{R}_{1}=\mathrm{OCH}_{3}, \mathrm{R}_{\mathbf{2}}=\mathrm{CH}_{3}$
Acacia kamerunensis: Separated phytochemicals having cytotoxic activity from this species are Kamerunoside A (43), Kamerunoside B (44), and Kamerunoside C (45) (Figure 5) (Tchoukoua et al., 2018).

Acacia nilotica: The elucidated chemical compounds from this species with antioxidant characteristics are Quercetin (11), Gallic acid (12), Catechin (21), Methyl gallate (51), Catechin 5-Ogallate (52), Gallocatechin-5-O-gallate (53), 1-Ogalloyl- $\beta$ - $D$-glucose $\quad(\mathbf{5 4}), \quad 1,6$-di-O-galloyl- $\beta$-Dglucose (55), Digallic acid (56), Acacetin (57), 1,2,3Benzenetriol (58), $\alpha, \beta$-glucooctanoic acid (59), Isoproposy-2-dimethyl-silyloxybenzene

(60), 
Proflavine (61), and Methyl 10-methyl-undecanoate 2017; Revathi et al., 2017).

(62) (Figure 7) (Salem et al., 2011; Rather et al.,

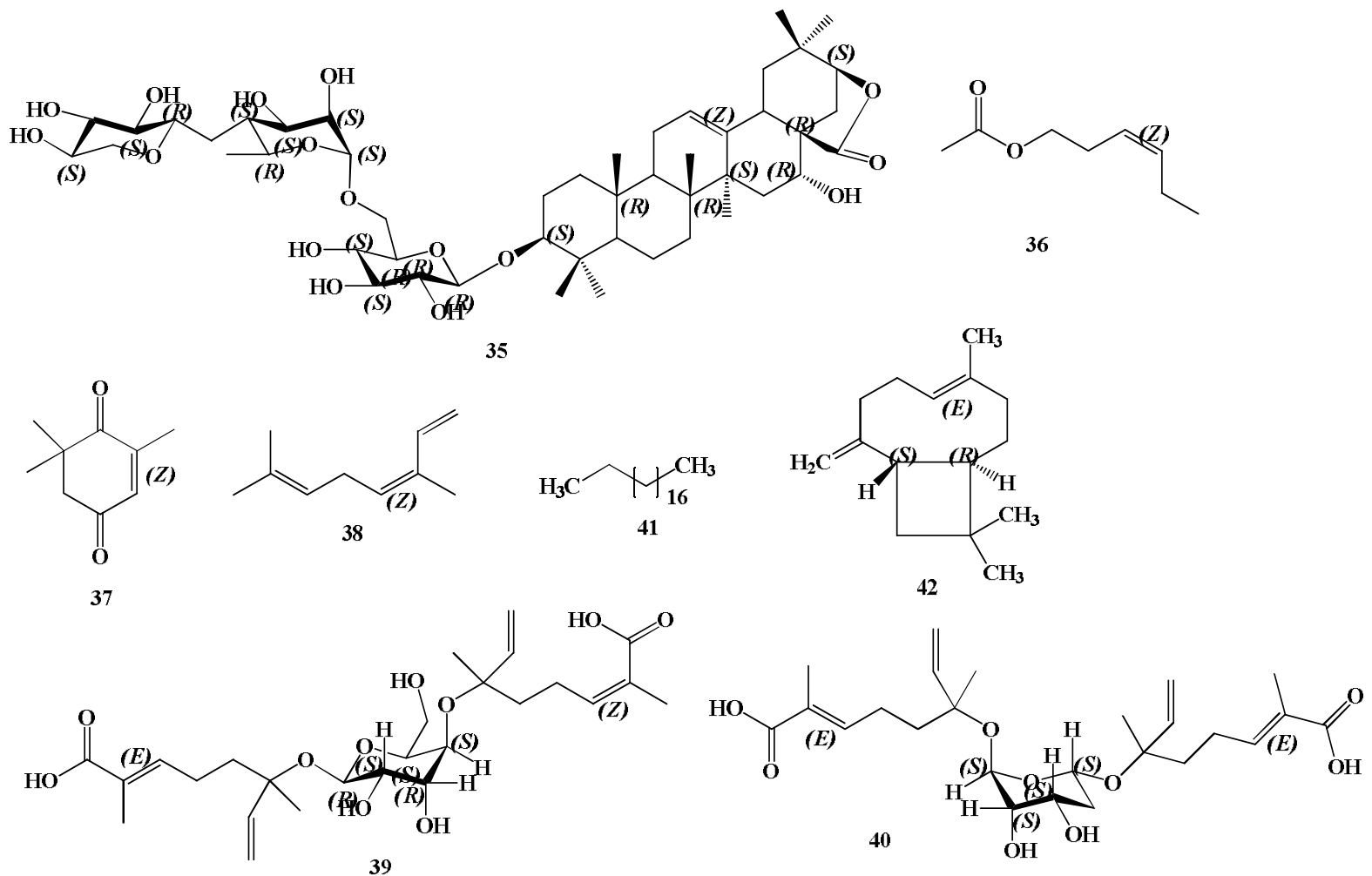

Figure 4. Molecules from Acacia cyclops.

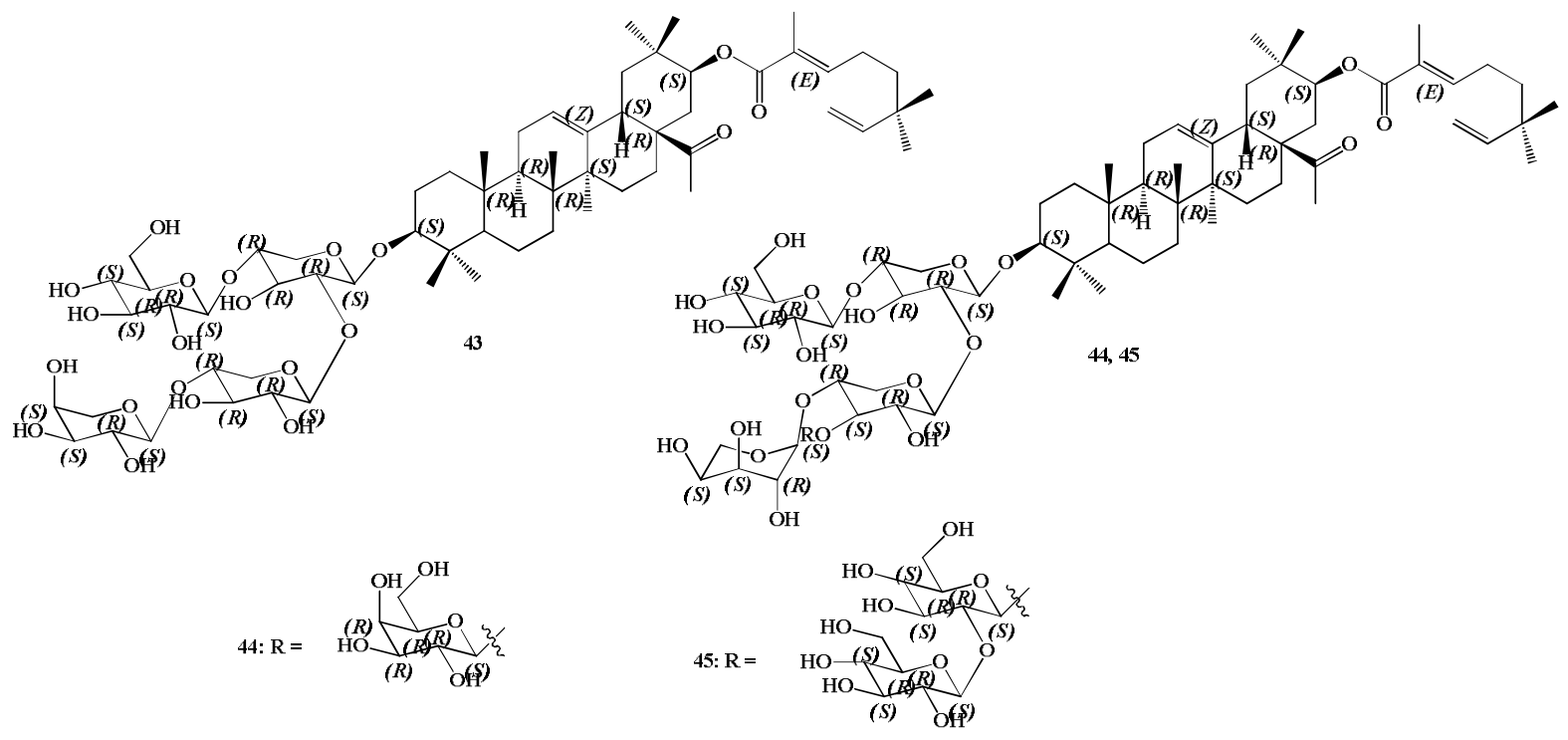

Figure 5. Triterpene saponins from Acacia kamerunensis. 
Acacia mearnsii: Elucidated chemical moieties from this medicinal plant are Catechin (21), Gallocatechin (46), Fisetinidol (47), Fisetinidol-4-ol (48), Robinetinidol (49), and Robinetinidol-4-ol (50).
The reported phytoconstituents showed antiinflammatory and carbolytic activity (Figure 6) (Jia et al., 2017).<smiles>[R]c1cc(-c2cc3c(O)cc(O)cc3o2)cc(O)c1O</smiles>

46: $\mathrm{R}_{1}=\mathrm{OH}$<smiles></smiles>

$$
\begin{aligned}
& \text { 47: } R_{1}=R_{2}=H \\
& \text { 48: } R_{1}=H, R_{2}=O H \\
& \text { 49: } R_{1}=O H, R_{2}=H \\
& 50: R_{1}=O H, R_{2}=O H
\end{aligned}
$$

Figure 6. Phytoconstituents from Acacia mearnsii.

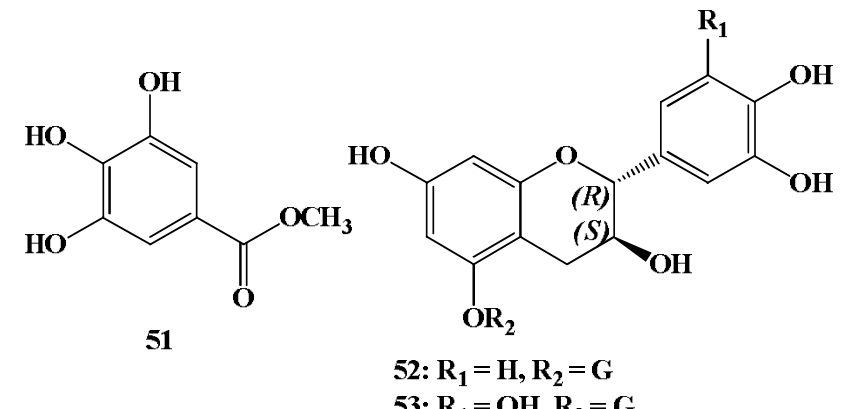
53: $\mathrm{R}_{1}=\mathrm{OH}, \mathrm{R}_{\mathbf{2}}=\mathrm{G}$

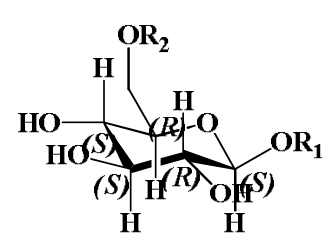
54: $\mathrm{R}_{\mathbf{1}}=\mathrm{G}, \mathrm{R}_{\mathbf{2}}=\mathrm{H}$ 55: $R_{1}=R_{2}=G$<smiles>[C-]C(=O)c1cc(O)c(O)c(O)c1</smiles><smiles>O=C(O)c1cc(O)c(OC(=O)c2cc(O)c(O)c(O)c2)c(O)c1</smiles><smiles>COc1ccc(-c2cc(=O)c3c(O)cc(O)cc3o2)cc1</smiles><smiles>Oc1cccc(O)c1O</smiles>

58<smiles>O=C1OC(C(O)C(O)C(O)CO)C(O)C1O</smiles><smiles>CC(C)Oc1ccccc1O[Si](C)(C)C(C)C</smiles><smiles>Nc1ccc2cc3ccc(N)cc3nc2c1</smiles>

61<smiles>COC(=O)C1OC(OC)C(O)C(O)C1=O</smiles>

Figure 7. Compounds from Acacia nilotica. 
Acacia oxyphylla: Isolated compound having anthelmintic property from this plant is 12-Amino7,17-dioxo-2-oxa-8,16-diazatricylo [14.2.2.23, 6] tetraicosa-1 (20),3,5,18,21,23-hexaene-12-carboxylic acid (63) (Figure 8) (Roya et al., 2012).<smiles>NC12CCCNC(=O)c3ccc(cc3)Oc3ccc(cc3)C(=O)NCCCC1(N)C(=O)O2</smiles>

Figure 8. Molecule from Acacia oxyphylla.

Acacia pennata: Derived flavonoid glycosides from this plant are 2R, 3S)-3,5,7-trihdyroxyflavan-3$O$ - $\alpha$-L-rhamnopyranoside (64), (2S)-5,7-dihydroxyflavan-7- $O-\beta-D$-glucopyranoside-(4a $\rightarrow 8)$-epiafzelechin-3- $O$-gallate $\quad(65), \quad(2 \mathrm{R})-4,7$-dihydroxyflavan$(4 \alpha \rightarrow 8)-(2 \mathrm{R}, 3 \mathrm{~S})-3, \quad 5,7$-trihdyroxyflavan-3-O- $\alpha-\mathrm{L}-$ rhamnopyranoside (66), 5,7-dihydroxyflavone-6-C$\beta$-boivinopyranosyl-7- $O$ - $\beta$ - $D$-glucopyranoside (67), 5,7-dihydroxyflavone 7-O- $\beta$ - $D$-glucopyranosyl-8-C$\beta$-boivinopyranoside (68), Quercetin-3-O- $\beta-D$-glucopyranoside (69) Quercetin-3-O- $\alpha$-L-rhamnopyranoside (70), Chrysin-7-O- $\beta-D$-glucopyraniside (71), 3-O- $\alpha$-L-Rhamnopyranoside (72), Koaburanin (73), and Pinocembrin-7- $O$ - $\beta$ - $D$-glucopyranoside (74) (Figure 9) (Kim et al., 2015).<smiles>[R]C1Cc2c(O)cc(O)cc2O[C@H]1c1ccccc1</smiles><smiles></smiles>

67: $R_{1}=$ Boiv, $R_{2}=O G l c, R_{3}=H$ 68: $R_{1}=I, R_{2}=O G l c, R_{3}=$ Boiv

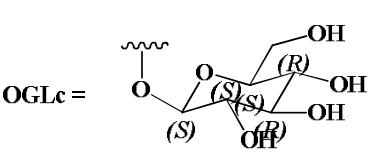

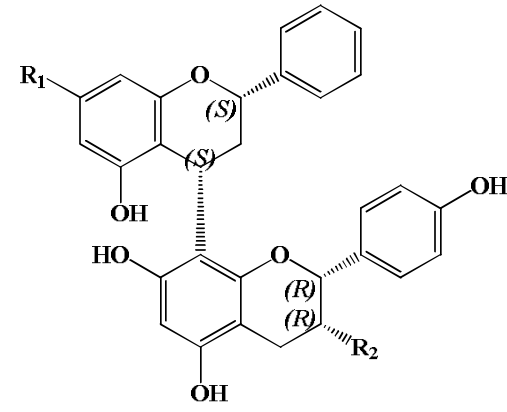
65: $\mathrm{R}_{1}=\mathrm{OGIc}, \mathrm{R}_{2}=$ Gall<smiles>[R2]c1cc(O)c2c(=O)c([R1])c(-c3cc([R4])c([R4])c([R3])c3)oc2c1</smiles>

69: $\mathrm{R}_{1}=\mathrm{OGIc}, \mathrm{R}_{2}=\mathrm{OH}, \mathrm{R}_{3}=\mathrm{OH}, \mathrm{R}_{4}=\mathrm{OH}, \mathrm{R}_{5}=\mathrm{H}$

70: $\mathrm{R}_{1}=\mathrm{ORha}, \mathrm{R}_{2}=\mathrm{OH}, \mathrm{R}_{3}=\mathrm{OH}, \mathrm{R}_{4}=\mathrm{OH}$

71: $\mathrm{R}_{1}=\mathrm{OII}, \mathrm{R}_{2}=\mathrm{OGIc}, \mathrm{R}_{3}=\mathrm{II}, \mathrm{R}_{4}=\mathrm{II}, \mathrm{R}_{5}=\mathrm{II}$

72: $\mathrm{R}_{1}=\mathrm{OGlc}, \mathrm{R}_{\mathbf{2}}=\mathrm{OH}, \mathrm{R}_{3}=\mathrm{H}, \mathrm{R}_{\mathbf{4}}=\mathrm{OH}, \mathrm{R}_{5}=\mathrm{II}$

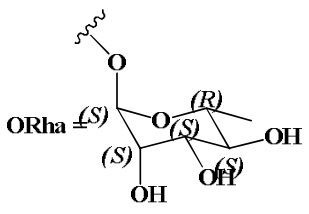

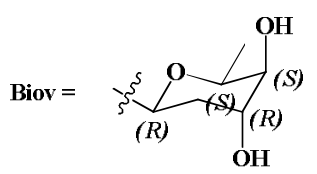<smiles>CCOC(=O)c1cc(O)c(O)c(O)c1</smiles>

Figure 9. Glycosides from Acacia pennata. 


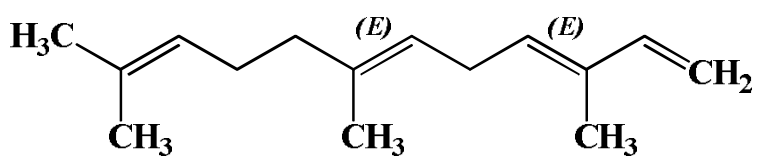

75

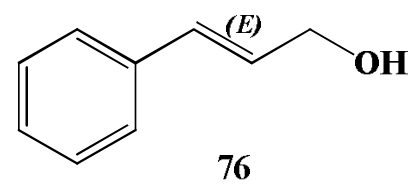

76

Figure 10. Constituents of essential oils from Acacia mollissima.

Acacia mollissima: Reported essential oil from Acacia mollissima are (E,E)- $\alpha$-Farnesene (75) and (E)-Cinnamyl alcohol (76) (Figure 10) (Jelassi et al., 2017).

\section{Conclusion}

Chemical compounds from ten species of Acacia have been studied and structurally different molecules were achieved from these species. This review revealed that Acacia can be a great source of secondary metabolites as well as pharmaceutical moieties.

\section{References}

Hussain, M.M. 2019. A comprehensive review on the phytoconstituents from six species of the genus Amaranthus. Bangladesh Pharm. J. 22, 117-124.

Hussain, M.M. 2018. A short review on phytoconstituents from the genera Albizzia and Erythrina. Bangladesh Pharm. J. 21, 160-172.

Hussain, M.M., Tuhin, M.T.H., Akter, F. and Rashid, M.A. 2016a. Constituents of Erythrina-a potential source of secondary metabolites: A review. Bangladesh Pharm. J. 19, 237-253.

Hussain, M.M., Tahia, F. and Rashid, M.A. $2016 \mathrm{~b}$. Secondary metabolites from some species of Albizzia: A review. Bangladesh Pharm. J. 19, 1-8.

Billah, A.H.M.M., Hussain, M.M., Dastagir, M.G., Ismail, M. and Quader, A. 2013. $\alpha$-Spinasterol from Amaranthus spinosus stem. Bol. Latinoam. Caribe Plant. Med. Aromat. 12, 15-17.

Hussain, M.M., Dastagir, M.G., Billah, A.H.M M. and Ismail, M., 2011. Alpinum isoflavone from Erythrina stricta Roxb. Bol. Latinoam. Caribe Plant. Med. Aromat. 10, 88-90.

Hussain, M.M., Mughul, M.M.R., Alam, M.M., Dastagir, M.G., Billah, A.H.M.M. and Ismail, M. 2010. Antimicrobial activity of $n$-hexane and ethyl acetate extracts of Erythrina stricta Roxb. Bangladesh J. Microbiol. 27, 65-66.
Ismail, M., Hussain, M.M., Dastagir, M.G., Billah, M. and Quader, A. 2010. Phytochemical and antimicrobial investigation of Luffa cylindrical. Bol. Latinoam. Caribe Plant. Med. Aromat. 9, 327-332.

Hussain, M.M., Rahman, M.S., Jabber, A. and Rashid, M. A. 2008. Phytochemical and biological investigation of Albizzia lebbek Benth. Bol. Latinoam. Caribe Plant. Med. Aromat. 7, 273-278.

Tchoukoua, A., Tabopda, T.K., Uesugi, S., Ohno, M., Kimura, K.I., Kwon, E., Momma, H., Horo, I., Caliskan, O.A. Shiono, Y. and Ngadjui, B.T. 2017. Triterpene saponins from the roots of Acacia albida Del. (Mimosaceae). Phytochem. 136, 31-38.

Li, X.C., Yang, L.X., Wang, H.Q. and Chen, R.Y. 2011. Phenolic compounds from the aqueous extract of Acacia catechu. Chinese Chem. Lett. 22, 1331-1334.

Castillo-Mitre, G.F., Olmedo-Juárez, A., Rojo-Rubio, R., González-Cortázar, M., Mendoza-de Gives, P., Hernández-Beteta, E.E., Reyes-Guerrero, D.E., ReyesGuerrero, M.E., López-Arellano, J.F., VázquezArmijoa, G. and Ramírez-Vargas, A. 2017. Zamilpa. Caffeoyl and coumaroyl derivatives from Acacia cochliacantha exhibit ovicidal activity against Haemonchus contortus. J. Ethnopharmacol. 204, 125131.

Jelassi, A., Ayeb-Zakhama, A.E., Nejma, A.B., Chaari, A., Harzallah-Skhiri, F. and Jannet, H.B. 2016. Phytochemical composition and allelopathic potential of three Tunisian Acacia species. Indus. Crops Products, 83, 339-345.

Tchoukoua, A., Tabopda, T.K., Usukhbayar, N., Ken-Ichi Kimura, E., Kwon, E., Momma, H., Koseki, T., Shiono, Y. and Ngadjuia, B.T. 2018. New triterpene saponins from the stem of Acacia kamerunensis (Mimosaceae). Phytochem. Lett. 23, 21-25.

Jia, X., Mary, G., Debora, E., Slavko, K., Fei, W. and Ann, L.M. 2017. Polyphenols isolated from Acacia mearnsii bark with anti-inflammatory and carbolytic enzyme inhibitory activities. Chinese J. Nat. Prod. 15, 0816-0824. 
Salem, M.M., Davidorf, F.H. and Abdel-Rahman, M.H. 2011. In vitro anti-veal melanoma activity of phenolic compounds from the Egyptian medicinal plant Acacia nilotica. Fitoterapia 82, 1279-1284.

Rather, L.J., Akhter, S., Padder, R.A., Hassan, Q.P., Hussain, M., Ali, M.A. and Mohammad, F. 2017. Colorful and semi durable antioxidant finish of woolen yarn with tannin rich extract of Acacia nilotica natural dye. Dyes Pigments 139, 812-819.

Revathi, S., Govindarajan, R.K., Rameshkumar, N., Hakkim F. L., Mohammed, A.B., Krishnan, M. and Kayalvizhia, N. 2017. Anti-cancer, anti-microbial and anti-oxidant properties of Acacia nilotica and their chemical profiling. Biocatal. Agri. Biotechnol. 11, 322-329.

Roya, B., Dasguptaa, S., Manivelb, V., Parameswaranc, P.S. and Giria, B.R. 2012. Surface topographical and ultra structural alterations of Raillietina echinobothrida and Ascaridia galli induced by a compound isolated from Acacia oxyphylla. Vet. Parasitol. 185, 322-326.

Kim, A., Choi, J., Htew, K.M., Chin, Y-W., Kim, J. and Yoon, K.D. 2015. Flavonoid glycosides from the aerial parts of Acacia pennata in Myanmar. Phytochemistry 118, 17-22.
Mohammed, M.M., Ali, A.A., Desoky, E-E.K. and Gobraeil, L.G. 2018. Compounds isolation and in vitro antioxidant activity evaluation of Faidherbia albida (Del.) A. Chev. Leaves ethanolic extract. J. Pharmacog. Phytochem. 7, 471-475.

Kotze, M.J., Jürgens, A., Johnson, S.D. and Hoffmann, J.H. 2010. Volatiles associated with different flower stages and leaves of Acacia cyclops and their potential role as host attractants for Dasineura dielsi (Diptera: Cecidomyiidae). South Afr. J. Bot. 76, 701-709.

Jelassi, A., Bergaoui, A.Z., Nejma, A.B., Belaiba, M., Bouajila, J. and Jannet, H.B. 2014. Two new unusual monoterpene acid glycosides from Acacia cyclops with potential cytotoxic activity. Bioorg. Med. Chem. Lett. 24, 3777-3781.

Jelassi, A., Hassine M., Hlila, M.B. and Jannet, H.B. 2017. Chemical composition, antioxidant properties, $\alpha$ glucosidase inhibitory and antimicrobial activity of essential oils from Acacia mollissima and Acacia cyclops cultivated in Tunisia. Chem. Biodiver. 14, e1700252. 